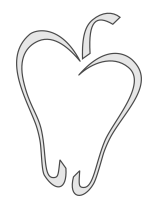

ISSN $1852-4249$

\title{
An improvement to the measurement of Dalitz plot parameters
}

\begin{abstract}
S. Ghosh, ${ }^{1 *}$ A. $\operatorname{Roy}^{1 \dagger}$
Precise measurement of the Dalitz plot parameters of the $\eta^{\prime} \rightarrow \eta \pi^{+} \pi^{-}$decay gives a better insight of the dynamics of the heavy pseudo-scalar meson. Various measurements of the Dalitz plot parameters of the $\eta^{\prime}$ meson have been performed with the detection of all the final state particles including the neutral decay of $\eta \rightarrow 2 \gamma$. In many experiments, reconstruction of the $\eta$ meson from the neutral decay modes comes with the disadvantage of poor resolution and low efficiency compared to that of the charged particles. In this article, a study of the Dalitz plot parameters keeping the $\eta$ meson as a missing particle is presented. The method is found to be advantageous in the case of poor photon resolution. Effect of the charged particle resolution on the Dalitz variable $\mathrm{Y}$ is also examined. This work may provide guidance to select a suitable method for the Dalitz plot analysis, depending on the detector resolution.
\end{abstract}

\section{Introduction}

Low energy quantum chromodynamics (QCD) is effectively studied in the framework of the chiral perturbation theory (ChPT). The Lagrangians for the hadronic processes are derived from ChPT and the effective degrees of freedom are, usually, the octet of pseudo-scalar mesons $(\pi, \mathrm{K}, \eta)[1]$. The observed mass of the $\eta^{\prime}$ meson in nature is much higher than the other pseudo-scalar mesons, which gives the axial $U(1)$ anomaly $[2,3]$. This suggests the possible existence of many unsolved problems that could provide inputs to the Lagrangian. Study of the production and decay of the $\eta^{\prime}$ meson is, therefore, important to both theory and experiment [4]. Some possible dynamics that could be studied in the $\eta^{\prime}$ decay model are the new sym-

\footnotetext{
*E-mail: phd12115113@iiti.ac.in

$\dagger$ E-mail: ankhi@iiti.ac.in

1 Discipline of Physics, School of Basic Sciences, Indian Institute of Technology Indore, Khandwa Road, Simrol, MP-453552, India.
}

metry and the symmetry breaking during interactions [5], gluonic contributions, gauge field configurations with non-zero winding number, and the quark instantons $[6,7]$. This has motivated many groups to study both charged and uncharged decays of the $\eta^{\prime}$ meson $[1,8]$.

The Dalitz plot plays an important role as a useful tool to study decay dynamics of a meson decaying into three bodies. As the three-body decay has two degrees of freedom, one can define two linearly independent variables to represent the decay in the phase space. In this article, we shall study the decay of $\eta^{\prime} \rightarrow \eta \pi^{+} \pi^{-}$and for that we define the Dalitz plot variables [9] as

$$
\begin{aligned}
& X=\frac{\sqrt{3}\left(T_{\pi^{+}}-T_{\pi^{-}}\right)}{Q}, \\
& Y=\frac{\left(m_{\eta}+2 m_{\pi}\right)}{m_{\pi}} \cdot \frac{T_{\eta}}{Q}-1 .
\end{aligned}
$$

Here $T$ and $m$ are, respectively, the kinetic energy (in the rest frame of $\eta^{\prime}$ ) and the mass of the particles indicated by the subscripts. Then, $\mathrm{Q}=T_{\pi^{+}}$ 
Papers in Physics, vol. 9, ART. 090009 (2017) / S. Ghosh et al.

$+T_{\pi^{+}}+T_{\pi^{+}}=m_{\eta^{\prime}}-m_{\eta}-2 m_{\pi}$, is the available energy of the reaction. The decay dynamics of the $\eta^{\prime}$ mesons is studied in the form of Dalitz plot parameters which are obtained by fitting the Dalitz plot with the following general parameterization:

$$
f(X, Y)=A\left(1+a Y+b Y^{2}+c X+d X^{2}\right) .
$$

Here, $a, b, c$, and $d$ are the Dalitz plot parameters of the decay and $A$ is the overall normalization constant. The measurement of Dalitz plot parameters are specifically important to understand and crosscheck the correct inputs to the theoretical distribution of the Lagrangian [10]. It is, therefore, important to measure the Dalitz plot parameters precisely with good statistics and resolution.

The Dalitz plot parameters of the $\eta^{\prime} \rightarrow \eta \pi^{+}$ $\pi^{-}$decay have been measured in the experiments of the VES [11] and the BESIII [12] collaborations. GAMS-4 $\pi$ [13] and IHEP-IISN-LANL-LAPP collaborations [7] have reported the Dalitz plot parameters for the neutral decay mode $\left(\eta \pi^{0} \pi^{0}\right)$ of the $\eta^{\prime}$ meson. The values of the Dalitz plot parameters for both of these decay modes of the $\eta^{\prime}$ meson should be the same under the isospin limit. However, this is not observed in the above experiments. This discrepancy may be reconciled by precise measurements of the small Dalitz plot parameters with high statistics. The previous experiments have identified the final state particles $\left(\pi^{+}, \pi^{-}\right.$and $\gamma)$ of the $\eta^{\prime} \rightarrow(\eta) \pi^{+} \pi^{-} \rightarrow(2 \gamma) \pi^{+} \pi^{-}$decay and used the invariant mass method for the Dalitz plot analysis. If the photon resolution is poor, the reconstruction efficiency of the $\eta$ meson will be significantly low and will also affect the Dalitz variable $\mathrm{Y}$ and the parameters related to this variable.

Here, we will be reporting a method that improves the Dalitz plot parameters in the measurements of the $\eta^{\prime}$ decay modes and found particularly advantageous in the case of poor photon resolution. We shall consider the production of $\eta^{\prime}$ meson through photo-production reaction first and then introduce the detector resolution to all the final state particles $\pi^{+}, \pi^{-}$, p and $\gamma$ of the channel $\gamma+\mathrm{p} \rightarrow \eta^{\prime} p \rightarrow(\eta) \pi^{+} \pi^{-} p \rightarrow(2 \gamma) \pi^{+} \pi^{-} \mathrm{p}$ to simulate the detector environment. The Dalitz plot parameters are then calculated using the following methods: (a) exclusive measurement or invariant mass method and (b) missing measurement method. The exclusive measurement method is commonly used, where all the final state particles $\left(\pi^{+}, \pi^{-}\right.$and $\left.\gamma\right)$ are considered and $\eta$ is reconstructed as the invariant mass $(\eta \rightarrow 2 \gamma)$. In the missing measurement method, we have reconstructed the $\eta^{\prime}$ meson as a missing particle using the information of the recoiled proton, the beam photon and the target proton $\left(\gamma_{\text {beam }}+p_{\text {target }} \rightarrow\right.$ $\left.\eta^{\prime} p_{\text {recoil }}\right)$. The $\eta^{\prime}$ information along with $\pi^{+}$and $\pi^{-}$is used to reconstruct the $\eta$ meson as a missing particle. The calculations of the Dalitz plot parameters using both these methods are described and compared. In addition to that, a bin width study is also performed with missing analysis to optimize the bin size for the extraction of the Dalitz plot parameters. In the missing measurement method, a dependence of the Dalitz variable $\mathrm{Y}$ on the detector resolutions of the charged particles has also been examined. Further, to study the effect of the background, method (b) was subjected to a combinatoric background channel and the Dalitz plot parameters are then systematically studied with varying background component in the mixture of the signal and the background. The background is then subtracted from every Dalitz plot bin and the parameters are reported.

\section{Model to fold the detector reso- lutions}

The Pluto simulation framework (version 5.42) developed by the HADES collaboration was used to generate hadronic physics reactions for this analysis [14]. Dalitz plot parameters of the $\eta^{\prime} \rightarrow \eta \pi^{+}$ $\pi^{-}$decay from the BESIII [12] experiment were used as input parameters for the generated events. A total of $10^{5} \gamma+\mathrm{p} \rightarrow \eta^{\prime} \mathrm{p}$ events, each with a photon beam energy of $2.5 \mathrm{GeV}$ were generated in the phase space model for this analysis. However, the energy of the $\eta^{\prime}$ meson is arbitrary as the Dalitz plot parameters are independent of the initial energy of the $\eta^{\prime}$ meson. The detector effects are absent in the events generated by the Pluto event generator. However, in reality, the momentum of the particle passing through the detector is modified according to the detector resolution. Generally, the detector response is distributed with a resolution which is dependent on the magnitude of the particle's momentum. To incorporate the detector response, each component of the momentum 

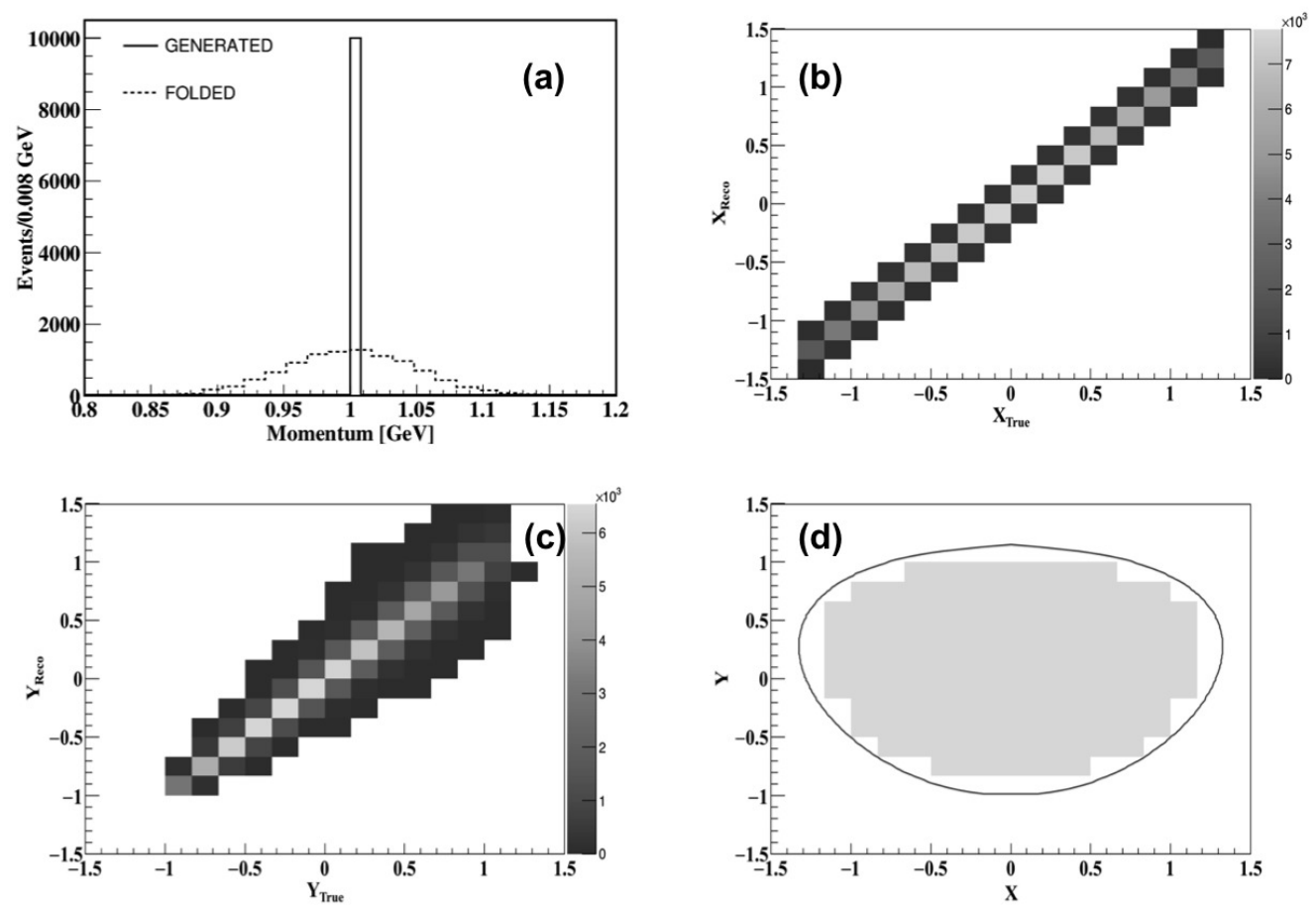

Figure 1: (a) Number of events as a function of the momentum, where the solid histogram is for the events with unit momentum before folding and the dashed histogram is for the events after the folding with a Gaussian distribution whose $\sigma$ is $3 \%$ of the mean. (b) Number of generated vs. reconstructed events in the Dalitz variable bins X. (c) Number of generated vs. reconstructed events in the Dalitz variable bins Y. (d) The Dalitz variable X vs. Y showing the bins completely inside the Dalitz plot boundary.

of the particles are convoluted with a random number sampled from a Gaussian distribution of mean 1 and $\sigma=3 \%$ of the mean, which introduces the momentum dependent resolution to the particle. This effect transforms particles with a single momentum into a particle with a momentum distribution as shown in Fig. 1(a) [15].

Since we are studying a case where the photon resolution is poor compared to the charged particle resolution, the final state charged particles $\left(\pi^{+}\right.$, $\pi^{-}$and $\mathrm{p}$ ) are folded with $1 \%$ resolution $[16,17]$ in the momentum components to simulate a detectorlike effect, whereas $6 \%$ resolution is used for the photons. There are experiments that suffer from poor photon resolution for which $6 \%$ is rather an underestimation [18]. Dalitz plot variables X and $\mathrm{Y}$, after the addition of resolution to the final state particles in momentum components, are compared with the generated Dalitz plot variables as shown in Fig. 1(b) and (c). It is observed in Fig. 1(c) that at higher values of $\mathrm{Y}$, the bin migration is more significant. The reason for this is that a higher value of $\mathrm{Y}$ corresponds to a high energy $\eta$ meson, which decays to energetic photons with poor resolution. This leads to a enhanced bin migration at higher value of $\mathrm{Y}$.

Folding has the effect of migrating the events from one bin to the neighboring bins of the Dalitz plot. It distributes the events in the bins away from the diagonal, creating a homogeneous migration of the events and thus allows to calculate the parameters from a properly binned Dalitz plot. Both Dalitz plot variables, shown in Fig. 1, are divided into 18 bins from -1.5 to 1.5 . Though the migration is observed for the individual Dalitz variables shown in Fig 1(b) and (c), the maximum number 
Papers in Physics, vol. 9, ART. 090009 (2017) / S. Ghosh et al.
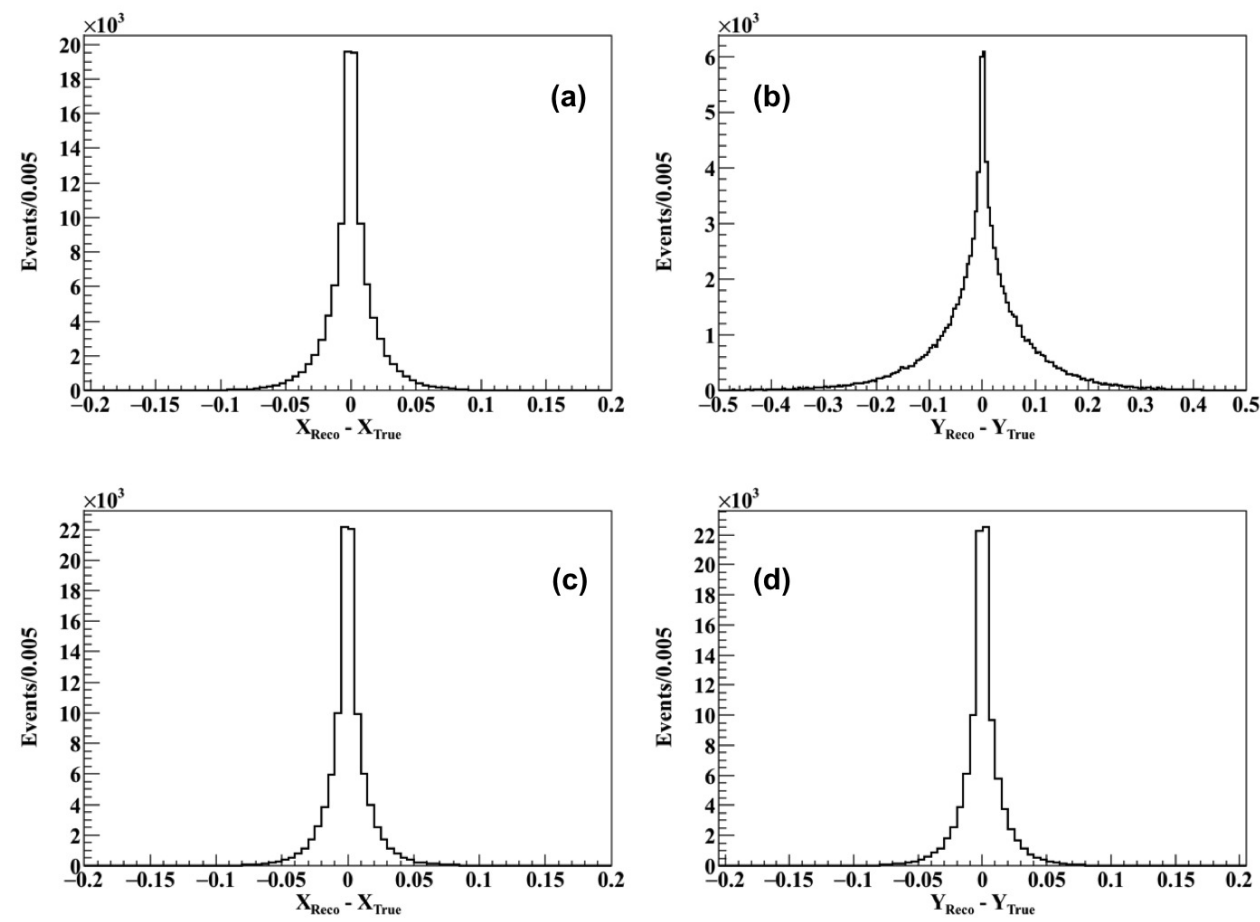

Figure 2: (a) Number of events vs. difference of the reconstructed (after folding) and the true value of the Dalitz variable $\mathrm{X}$ and (b) the Dalitz variable $\mathrm{Y}$ for the exclusive measurement method. (c) Number of events vs. difference of the reconstructed and the true value of Dalitz variable X and (d) the Dalitz variable $\mathrm{Y}$ for the missing measurement method (b).

of events, however, lie on the diagonal and confined well within the resolution of the bins.

The general parameterization of Eq. (2) was used to parametrize the decay. A least square fitting procedure MINUIT [19] was used to minimize the $\chi^{2}$ given in Eq. (3) to fit each bin of the Dalitz plot.

$$
\chi^{2}=\sum\left(\frac{N_{j}-f\left(X_{j}, Y_{j}\right)}{\Delta N_{j}}\right)^{2} .
$$

Where $N_{j}$ and $\Delta N_{j}$ denotes the number of events and their statistical uncertainties for the Dalitz plot bin $j(j=1,2, \ldots, n) . X_{j}$ and $Y_{j}$ are the central coordinates of each bin, and $f\left(X_{j}, Y_{j}\right)$ denotes the fitted form of the polynomial. The bins on the boundary of the Dalitz plot are removed and only bins which are completely inside the boundary are considered as shown in Fig. 1(d).

\section{i. Method (a): Exclusive measurement}

In this method, measurement of the Dalitz plot parameters are performed with all the detected final state particles $\pi^{+}, \pi^{-}$and $\gamma$. To calculate the resolution of Dalitz variables X and Y, first a difference of the reconstructed (after folding) and the true value of the Dalitz variable is considered. Then, the standard deviation of $\mathrm{X}$ and $\mathrm{Y}$ for a large number of events is calculated using

$$
\sigma=\sqrt{\left(\frac{\sum(x-\mu)^{2}}{N}\right)},
$$

where $x$ represents each value of the Dalitz variables and $\mu$ is the mean, which should be zero in this case, and $N$ is the total number of events. As seen from Fig. 2(a) and (b), the resolutions of the Dalitz variables X and Y are respectively 0.019 and 0.099, after the folding in the momentum compo- 
Papers in Physics, vol. 9, ART. 090009 (2017) / S. Ghosh et al.

Table 1: Dalitz plot parameters with varying number of bins in the Dalitz variables X and Y for methods (a) and (b).

\begin{tabular}{l|ccc|ccc}
\hline \hline & \multicolumn{3}{|c|}{ Method (a) } & \multicolumn{3}{c}{ Method (b) } \\
\hline Par & $17 \times 17$ & $18 \times 18$ & $19 \times 19$ & $17 \times 17$ & $18 \times 18$ & $19 \times 19$ \\
\hline$a$ & $-0.067 \pm 0.008$ & $-0.062 \pm 0.008$ & $-0.066 \pm 0.008$ & $-0.045 \pm 0.008$ & $-0.044 \pm 0.008$ & $-0.044 \pm 0.008$ \\
$b$ & $-0.134 \pm 0.015$ & $-0.142 \pm 0.015$ & $-0.145 \pm 0.014$ & $-0.058 \pm 0.016$ & $-0.063 \pm 0.015$ & $-0.081 \pm 0.015$ \\
$c$ & $0.014 \pm 0.005$ & $0.014 \pm 0.005$ & $0.009 \pm 0.005$ & $0.014 \pm 0.006$ & $0.014 \pm 0.005$ & $0.009 \pm 0.006$ \\
$d$ & $-0.098 \pm 0.009$ & $-0.096 \pm 0.009$ & $-0.093 \pm 0.009$ & $-0.085 \pm 0.010$ & $-0.081 \pm 0.009$ & $-0.092 \pm 0.009$ \\
\hline$\frac{\chi^{2}}{n d f}$ & 1.25 & 1.04 & 1.09 & 1.08 & 1.14 & 0.95 \\
\hline \hline
\end{tabular}

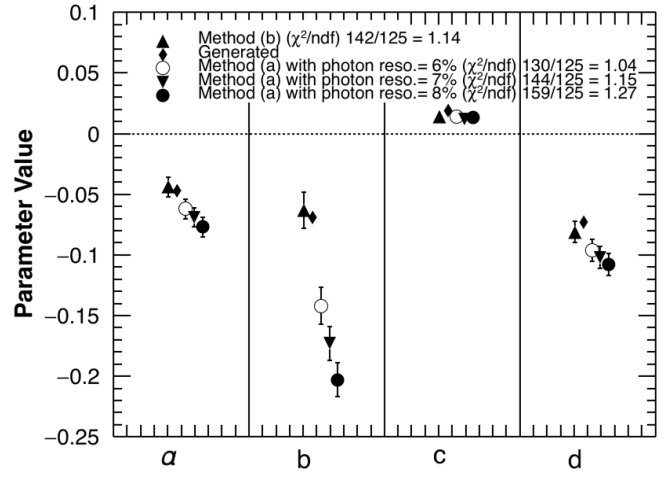

Figure 3: Comparison of the Dalitz plot parameters in an exclusive measurement method with varying photon resolution and missing measurement method along with the generated BESIII parameters.

nents. The resolution of the variables $\mathrm{X}$ and $\mathrm{Y}$ are independent in this method as the former was calculated from $\pi^{+}$and $\pi^{-}$but the later was calculated from the photons and, therefore, causing the resolution to be poor.

\section{ii. Method (b): Missing measurement}

In the missing measurement method, the Dalitz variables $\mathrm{X}$ and $\mathrm{Y}$ are calculated without using the information of the final photons from the decay of the $\eta$ meson. Only $\pi^{+}$and $\pi^{-}$are folded with the resolutions and the produced $\eta^{\prime}$ information is used to calculate the Dalitz variables $\mathrm{X}$ and $\mathrm{Y}$. The calculated resolutions of the Dalitz variables $\mathrm{X}$ and $\mathrm{Y}$ are both 0.016 and are shown in Fig. 2(c) and (d). In contrast to the exclusive measurement method, in this method, the resolutions of $\mathrm{X}$ and $\mathrm{Y}$ are same.

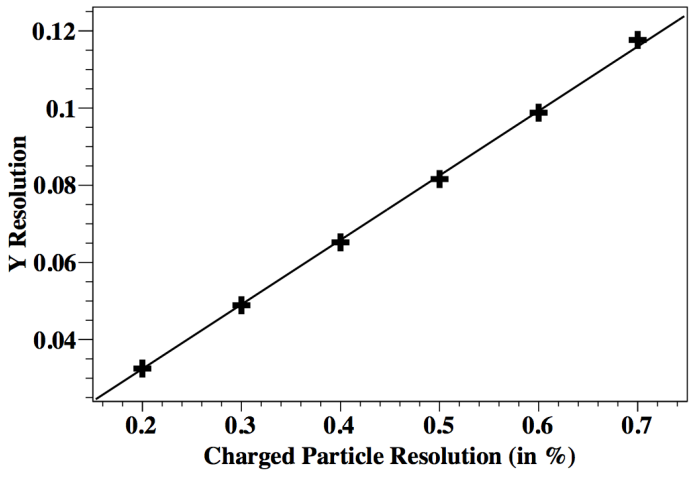

Figure 4: Variation of the $\mathrm{Y}$ resolution with the charged particle resolution.

This is due to the fact that both $\mathrm{X}$ and $\mathrm{Y}$ use the information of the same particles $\left(\pi^{+}\right.$and $\left.\pi^{-}\right)$with added resolution [20].

\section{iii. Comparison of the method (a) and (b)}

To compare the methods described above, we have extracted the Dalitz parameters by fitting the Dalitz plot with the parameterization defined in Eq. (2). All the bins are fitted to the general parameterization with the $\chi^{2}$ minimization. To find an optimized binning, the Dalitz plot parameters are calculated using the method (a) and (b) for varying number of bins in $\mathrm{X}$ and $\mathrm{Y}$ axis as shown in Table 1 . It is found that the best $\chi^{2} / \mathrm{ndf}$ is obtained for $10^{5}$ events with a binning of $18 \times 18$ for both methods. It can be seen that a systematics arises due to varying bin size on the parameters $a$ and $b$ in the invariant mass method. In both the methods a binning of $18 \times 18$ is thus used to calculate the Dalitz plot parameters, which is higher 
Papers in Physics, vol. 9, ART. 090009 (2017) / S. Ghosh et al.

than the resolution of the Dalitz variables $\mathrm{X}$ and Y. A comparison of the parameters obtained using the methods (a), with different photon resolution, and (b), with the generated BESIII parameters, is shown in Fig. 3 along with their errors and $\chi^{2} /$ ndf. It can be seen from the figure that with poorer photon resolution the parameters $a$ and $b$ systematically deviate from the generated value. Though the values of the parameters $c$ and $d$ are consistent as shown in Table 1, it is observed that due to poor photon resolution, deviation of the $a$ and $b$ parameters from the input in the exclusive measurement method is significant compared to the missing $2 \gamma$ measurement method. Even the deviation of parameter $b$ is higher than that of $a$, as $b$ is the coefficient of a quadratic $Y$. The poor photon resolution directly affects the $\eta$ momentum resolution, which is reflected in the poor $\mathrm{Y}$ resolution. The missing measurement method is, therefore, preferable for the Dalitz plot analysis in this case of poor photon resolution.

Since in the missing measurement method the Dalitz variable $\mathrm{Y}$ depends on the charge particle resolution, we have carried out another study to examine the variation of the $\mathrm{Y}$ resolution with the charged particle resolution. We found that $\mathrm{Y}$ varies linearly with the charged particle resolution as shown in Fig. 4. It can be concluded that if the charge particle resolution is equally poor as the photon resolution, the missing analysis method is still a better choice compared to the exclusive measurement method. In the present experimental condition of $1 \%$ charged particle resolution and $6 \%$ neutral particle resolution, the exclusive measurement method introduces a poorer Y resolution compared to the missing measurement method.

\section{Background study}

The missing measurement method comes with a cost of additional combinatoric background for the channel $\eta^{\prime} \rightarrow \eta \pi^{+} \pi^{-}$. This combinatoric background arises from $\pi^{+}$and $\pi^{-}$produced in the decays $\eta^{\prime} \rightarrow \eta \pi^{+} \pi^{-}$and $\eta \rightarrow \pi^{+} \pi^{-} \pi^{0}$, which leaves the $\pi^{+}$and $\pi^{-}$in both decays with a similar available energy and hence similar kinematics. We consider a background $\eta^{\prime} \rightarrow \eta \pi^{0} \pi^{0}$ generated with the same Dalitz plot parameters because of isospin symmetry [1] as $\eta^{\prime} \rightarrow \eta \pi^{+} \pi^{-}$and $\eta$ is further

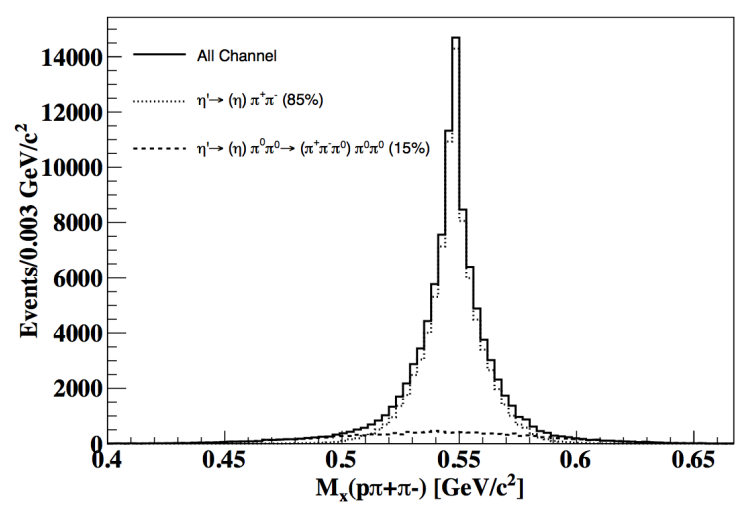

Figure 5: The number of events vs. the missing mass $M_{x}\left(\eta^{\prime}, \pi+\pi-\right)$ from the signal and background channel.

decayed into $\eta \rightarrow \pi^{+} \pi^{-} \pi^{0}$. We mixed the signal channel with 5\%,10\% and 15\% background and then folded it to add resolution. The Dalitz plot parameters from these three set are given in Table 2 . The missing mass $M_{x}\left(\eta^{\prime}, \pi+\pi-\right)$ in Fig. 5 clearly shows the contribution of the background channel in the $\eta$ meson mass region due to misidentification. The Dalitz plot parameters $a$ and $b$ related to the Dalitz variable Y are systematically shifted from their generated values due to the presence of this background. Meanwhile, the Dalitz parameters $c$ and $d$ do not change since $\pi^{+}$and $\pi^{-}$from both channels have similar kinematics, as shown in Fig. 6. However, this combinatoric background can be subtracted by determining the number of background events in each bin of the Dalitz plot. The contribution of background in each bin is calculated from the generated Monte-Carlo samples before implementing the resolution to the particles, which introduces a systematics from the migration of background events to the nearby bins in the Dalitz plot. The general parameterization of Eq. (2) is fitted to the background subtracted Dalitz plot and the parameters are given in Table 3. The systematics from the migration of background events is small in the missing measurement method, and the extracted parameters after background subtraction show that method (b) is a better choice than the exclusive measurement [method (a)] when charge particle resolution is $1 \%$ and photon resolution is $6 \%$. 
Papers in Physics, vol. 9, ART. 090009 (2017) / S. Ghosh et al.

Table 2: Comparison of the Dalitz plot parameters extracted from method (b) with different background contributions.

\begin{tabular}{ccccc}
\hline input pars & method (b) (No bkg) & method (b) (5\% bkg) & method (b) (10\% bkg) & method (b) (15\% bkg) \\
\hline$a(-0.047)$ & $-0.044 \pm 0.008$ & $-0.074 \pm 0.008$ & $-0.087 \pm 0.008$ & $-0.125 \pm 0.008$ \\
$b(-0.069)$ & $-0.063 \pm 0.015$ & $-0.078 \pm 0.015$ & $-0.130 \pm 0.015$ & $-0.119 \pm 0.015$ \\
$c(0.019)$ & $0.014 \pm 0.005$ & $0.016 \pm 0.005$ & $0.020 \pm 0.005$ & $0.015 \pm 0.005$ \\
$d(-0.073)$ & $-0.081 \pm 0.009$ & $-0.070 \pm 0.009$ & $-0.064 \pm 0.009$ & $-0.062 \pm 0.009$ \\
\hline$\frac{\chi^{2}}{n d f}$ & 1.14 & 0.86 & 0.94 & 1.16 \\
\hline \hline
\end{tabular}

Table 3: Comparison of the Dalitz plot parameters extracted from method (a) and the background subtracted Dalitz plot obtained from method (b).

\begin{tabular}{ccccc}
\hline \hline input pars & method (a) & method (b) (5\% bkg) & method (b) (10\% bkg) & method (b) $(15 \% \mathrm{bkg})$ \\
\hline$a(-0.047)$ & $-0.062 \pm 0.008$ & $-0.045 \pm 0.008$ & $-0.045 \pm 0.008$ & $-0.042 \pm 0.008$ \\
$b(-0.069)$ & $-0.142 \pm 0.015$ & $-0.066 \pm 0.016$ & $-0.086 \pm 0.016$ & $-0.084 \pm 0.017$ \\
$c(+0.019)$ & $0.014 \pm 0.005$ & $+0.018 \pm 0.006$ & $+0.022 \pm 0.006$ & $+0.019 \pm 0.006$ \\
$d(-0.073)$ & $-0.096 \pm 0.009$ & $-0.073 \pm 0.010$ & $-0.060 \pm 0.010$ & $-0.076 \pm 0.010$ \\
\hline$\frac{\chi^{2}}{n d f}$ & 1.04 & 0.93 & 1.15 & 1.02 \\
\hline \hline
\end{tabular}

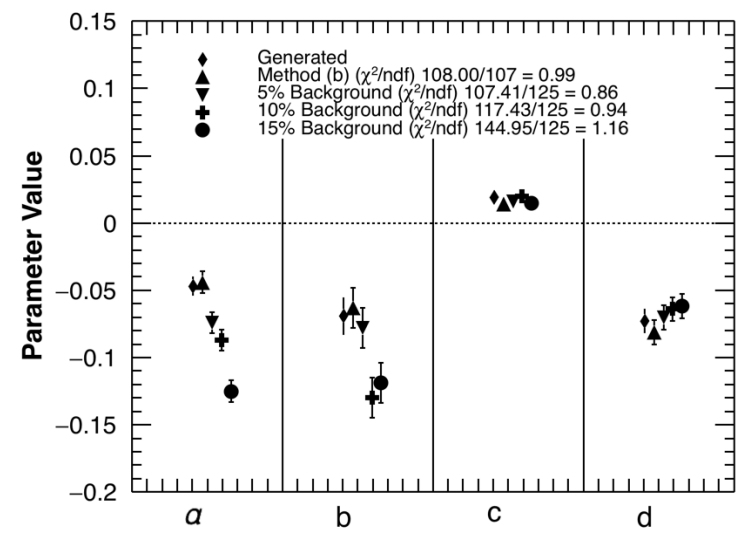

Figure 6: Comparison of the Dalitz plot parameters from generated and missing mass method without any background and with the background added in a proportion of $5 \%, 10 \%$ and $15 \%$ of the total number of events.

\section{Conclusions}

In this article, we have described two methods for measuring the Dalitz variables $\mathrm{X}$ and $\mathrm{Y}$ in the context of the decay $\eta^{\prime} \rightarrow \eta(2 \gamma) \pi^{+} \pi^{-}$. The exclusive measurement method and the invariant mass method, though commonly used for measuring the Dalitz variables, are shown here to be unsuitable in the case of poor photon resolution. In contrast, the missing $(2 \gamma)$ analysis method is found to be more suitable when the photon resolution is poor. The resolution in $\mathrm{Y}$ versus charge particle resolution, in the missing analysis method, suggests that, even in the case of equally poor charged particle resolution and photon resolution the missing analysis method is a better choice compared to the exclusive measurement method. The missing analysis method, however, comes with the disadvantage of the combinatoric background and the Dalitz plot parameters deviate systematically from their central values depending on the fraction of background present. Once the background subtraction is implemented, the missing analysis method performs even better and becomes more attractive. In conclusion, this work provides guidance to select a suitable method for the extraction of the Dalitz plot parameters depending on the detector resolution as well as the background present in the signal region and may be extended to the three body decay channels of other mesons when the detector resolution is poor.

[1] B Borasoy, R Nißler, Hadronic $\eta$ and $\eta^{\prime}$ decays, Eur. Phys. J. A 26, 383 (2005). 
Papers in Physics, vol. 9, ART. 090009 (2017) / S. Ghosh et al.

[2] K Naito, M Oka, M Takizawa, T Umekawa, $U_{A}(1)$ breaking effects on the light scalar meson spectrum, Prog. Theor. Phys. 109, 969 (2003).

[3] S Weinberg, The U(1) problem, Phys. Rev. D 11, 3583 (1975).

[4] J Bijnens, $\eta$ and $\eta^{\prime}$ physics, Proceedings of the 11th International Conference on MesonNucleon Physics and the Structure of the Nucleon, eConf C 070910, 104 (2007).

[5] N Beisert, B Borasoy, The $\eta^{\prime} \rightarrow \eta \pi \pi$ decay in $U(3)$ chiral perturbation theory, Nuc. Phys. A 705, 433 (2002).

[6] S D Bass, Gluonic effects in $\eta$ - and $\eta^{\prime}$-nucleon and nucleus interactions, Acta Phys. Slovaca 56, 245 (2006).

[7] D Alde et al., Matrix element of the $\eta^{\prime}$ (958) $\rightarrow \eta \pi^{0} \pi^{0}$ decay, Phys. Lett. B 177, 115 (1986).

[8] A Kupsc, Decays of $\eta$ and $\eta^{\prime}$ mesons: An introduction, Int. J. Mod. Phys. E 18, 1255 (2009).

[9] A H Fariborz, J Schechter, $\eta^{\prime} \rightarrow \eta \pi \pi$ decay as a probe of a possible lowest lying scalar nonet, Phys. Rev. D 60, 034002 (1999).

[10] R Escribano, P Masjuan, J J Sans-Cillero, Chiral dynamics predictions for $\eta^{\prime} \rightarrow \eta \pi \pi$, J. High Energy Phys. 2011, 094 (2011).

[11] V Dorofeev et al., Study of $\eta^{\prime} \rightarrow \eta \pi^{+} \pi^{-}$Dalitz plot, Phys. Lett. B 651, 22 (2007).
[12] M Ablikim et al., Measurement of the matrix element for the decay $\eta^{\prime} \rightarrow \eta \pi^{+} \pi^{-}$, Phys. Rev. D 83, 012003 (2011).

[13] A M Blik et al., Measurement of the matrix element for the decay $\eta^{\prime} \rightarrow \eta \pi^{0} \pi^{0}$ with the GAMS- $4 \pi$ spectrometer, Phys. Atom. Nucl.+ 72, 231 (2009).

[14] I Fröhlich et al., Pluto: A Monte Carlo simulation tool for hadronic physics, PoS(ACAT2007) 076, (2007).

[15] P Garg, D K Mishra, P K Netrakanti, A K Mohanty, B Mohanty, Unfolding of event-byevent net-charge distributions in heavy-ion collisions, J. Phys. G: Nucl. Part. Phys. 40, 055103 (2013).

[16] M Ullrich, W Khn, Y Liang, B Spruck, M Werner, Simulation of the BESIII endcap time of flight upgrade, Nucl. Instrum. Meth. A 769, 32 (2015).

[17] M Battaglieri, R De Vita, V Kubarovsky, Pentaquark at JLab: The g11 experiment in CLAS, AIP Conf. Proc. 792, 742 (2005).

[18] M Amarian et al., The CLAS forward electromagnetic calorimeter, Nucl. Instrum. Meth. A 460, 239 (2001).

[19] R Brun, F Rademakers, ROOT: An object oriented data analysis framework, Nucl. Instrum. Meth. A 389, 81 (1997).

[20] S Ghosh, Dalitz plot analysis of $\eta^{\prime} \rightarrow \eta \pi^{+} \pi^{-}$, AIP Conf. Proc. 1735, 030018 (2016). 